\title{
Dioscin attenuates oxLDL uptake and the inflammatory reaction of dendritic cells under high glucose conditions by blocking p38 MAPK
}

\author{
YING LI, YONG LI, TE YANG and MING WANG \\ Cardiovascular Department, Chongqing Traditional Chinese Medicine Hospital, Chongqing 400011, P.R. China
}

Received April 8, 2019; Accepted October 15, 2019

DOI: $10.3892 / \mathrm{mmr} .2019 .10806$

\begin{abstract}
Dioscin has been shown to affect the regulation of metabolic diseases, including diabetes; however, the mechanism of action is still unclear. Under high glucose (HG) conditions, the expression of scavenger receptors and the uptake of oxidized low-density lipoprotein (oxLDL) are upregulated in dendritic cells (DCs), which are critical steps in atherogenesis and inflammation. In this study, the focus was on the impact of dioscin on the function of DCs. Immature DCs were cultured with: $5.5 \mathrm{mM}$ glucose medium (control group); $30 \mathrm{mM}$ glucose medium (HG group); $\mathrm{HG}+10 \mathrm{mM}$ dioscin; $\mathrm{HG}+20 \mathrm{mM}$ dioscin; $\mathrm{HG}+30 \mathrm{mM}$ dioscin; and $\mathrm{HG}+40 \mathrm{mM}$ dioscin. For subsequent experiments, $30 \mathrm{mM}$ dioscin was used as the experimental concentration. Dichlorodihydrofluorescein fluorescence was used to measure the intracellular production of reactive oxygen species (ROS) in DCs. The expression levels of the scavenger receptors, including class A scavenger receptors (SR-A), CD36 and lectin-like oxidized low-density lipoprotein receptor-1 (LOX-1) were determined via quantitative PCR. The protein expression of p38 mitogen-activated protein kinase (MAPK) was determined by western blotting. Furthermore, ELISA was used to detect the levels of interleukin (IL)-6, IL-10 and IL-12. Finally, DCs were incubated with diOlistic (Dil)-labeled oxLDL, and flow cytometry analysis was used to investigate the Dil-oxLDL-incorporated fraction. The incubation of DCs with dioscin inhibited the induction of ROS production, in a dose-dependent manner, under HG conditions. The upregulation of SR-A, CD36 and LOX-1 genes was partially abolished by dioscin, which also partially reversed p38 MAPK protein upregulation. Furthermore, increased secretion of IL- 6 and IL-12, and decreased secretion of IL-10 in DCs, induced by HG, was also reversed by dioscin.
\end{abstract}

Correspondence to: Professor Ming Wang, Cardiovascular Department, Chongqing Traditional Chinese Medicine Hospital, 6 Pan Xi Bypass Road, Jiangbei, Chongqing 400011, P.R. China E-mail: xfeng_wang@126.com

Key words: dioscin, dendritic cell, scavenger receptors, type 2 diabetes, oxidized low-density lipoprotein, reactive oxygen species, atherosclerosis, immune response
To conclude, dioscin could attenuate the production of ROS, inflammatory cytokine secretion and oxLDL uptake by DCs in $\mathrm{HG}$ conditions by preventing the expression of scavenger receptors and p38 MAPK, thus playing a positive role in preventing atherogenesis.

\section{Introduction}

The prevalence of diabetes mellitus has increased substantially in the past decade, with the number of diabetes cases reaching 387 million $(8.3 \%)$ in 2014 globally $(1,2)$. In type 2 diabetes, the rates of microvascular and macrovascular complications are 18.8 and $12.7 \%$, respectively (3). Arteriosclerosis is the basis of vascular complications, and high glucose (HG) is the main factor in the development of arteriosclerosis $(4,5)$. During the course of arteriosclerosis development, dendritic cells (DCs) play a key role; DCs move towards the vessel wall, and recognize autoantigens and oxidized low-density lipoproteins (oxLDLs) (6). oxLDL uptake by DCs may enhance the presentation of lipid and peptide antigens to natural killer $\mathrm{T}$ cells and $\mathrm{T}$ cells to further stimulate vascular inflammation and monocyte adhesion in the atherosclerotic plaque $(7,8)$.

In DCs and intraperitoneal macrophages derived from diabetic mice, hyperglycemia increased reactive oxygen species (ROS) production $(5,9)$. Mitogen-activated protein kinase (MAPK) is an important protein kinase activator and a promising target in the treatment of diabetes, obesity and cardiovascular diseases, due to its important roles in cell growth, cell differentiation and energy metabolism (10). HG can increase the amount of ROS, and this can induce hyperactivation of p38 MAPK in DCs, leading to oxLDL uptake by DCs and atherosclerosis $(8,11,12)$.

Dioscin is a natural steroidal saponin that is isolated from certain medicinal plants or vegetables. Dioscin has numerous pharmacological actions, including anti-inflammatory, immunoregulatory, hypolipidemic, antiviral, antifungal and antitumor effects $(13,14)$. Dioscin has been shown to have protective effects in liver, kidney and lung damage/cancers through various mechanisms. For example, dioscin can activate the Notch1/Jagged1 pathway to promote hepatoproliferation, thus facilitating liver regeneration after partial hepatectomy (15). Dioscin showed a protective effect against thioacetamide-induced acute liver injuries in rats and mice, possibly via the inhibition of oxidative stress and inflammation 
due to its action on the farnesoid $\mathrm{X}$ receptor/AMP-activated protein kinase (AMPK) signaling pathway (16). Dioscin has been found to also prevent nonalcoholic fatty liver disease by adjusting lipid metabolism via the sirtuin 1 (Sirt1)/AMPK signal pathway (17). Additionally, dioscin exhibited a protective effect against lipopolysaccharide-induced liver injury and acute lung injury via the alteration of the Toll-like receptor $4 /$ myelid differentiation primary response 88 pathway $(18,19)$. In vitro dioscin attenuated cell damage and decreased renal injury in rats and mice treated with cisplatin through the microRNA-(miR)-34a/Sirt1 signaling pathway (20). During intestinal ischemia/reperfusion (II/R) injury, dioscin upregulated MAPK13 expression by decreasing miR-351-5p levels to inhibit inflammation and apoptosis, thus showing a protective effect (21). Dioscin exerted efficient anti-prostate cancer activity via activation of the estrogen receptor- $\beta$ (22). Dioscin showed anti-pancreatic cancer effects via miR-149-3P-mediated inhibition of the Akt1 signaling pathway (23). In addition, previous studies have suggested that dioscin also has beneficial effects in the regulation of metabolic diseases, such as diabetes, osteoporosis, obesity and hyperuricemia (24-27). However, the mechanism by which dioscin regulates diabetes is still unclear. Since DCs have critical roles in arteriosclerosis development, this study focused on the impact of dioscin on the function of DCs.

\section{Materials and methods}

Generation of monocyte-derived DCs. Peripheral blood mononuclear cells were obtained from 9 healthy volunteers in the Chongqing Traditional Chinese Medicine Hospital between 1 March, 2018 and 30 September, 2018 (6 males and 3 females aged 26-35 years). Mononuclear cells were layered in lymphocyte separation solution and centrifuged for $20 \mathrm{~min}$ at $800 \mathrm{x} \mathrm{g}$ using discontinuity density gradient centrifugation at room temperature. The interface was recovered and washed three times with Hank's solution and centrifuged three times at $600 \mathrm{x}$ g for $8 \mathrm{~min}$ at room temperature. The cells were magnetically sorted for CD14 after gradient centrifugation, adjusted to 5x10 $/ 1$ using RPMI 1640 medium (Hyclone; GE Healthcare Life Sciences) and incubated at $37^{\circ} \mathrm{C}$ in an atmosphere of $5 \% \mathrm{CO}_{2}$ for $2 \mathrm{~h}$. The non-adherent cells were removed, and the remaining adherent cells were incubated in $0.9 \mathrm{ml}$ RPMI 1640 medium supplemented with $0.1 \mathrm{ml}$ calf serum (Hyclone; GE Healthcare Life Sciences), $10 \mu 150 \mathrm{mg} / 1$ recombinant human granulocyte-macrophage colony-stimulating factor (PeproTech, Inc.) and $44 \mu 1$ recombinant human interleukin (IL)-4 (20 mg/l) (PeproTech, Inc.) at $37^{\circ} \mathrm{C}$ and an atmosphere of $5 \% \mathrm{CO}_{2}$. Immature DCs were harvested on day 6 . Then, cells were exposed to glucose $(5.5$ and $30 \mathrm{mM})$ or dioscin at various concentrations $(10,20,30$ and $40 \mathrm{mM})$ with $30 \mathrm{mM}$ $\mathrm{HG}$ for an additional $24 \mathrm{~h}$ (dioscin was purchased from Selleck Chemicals, with a purity of $>97 \%$ and a molecular weight of $869.04 \mathrm{~g} / \mathrm{mol}$ ). The cells were further incubated for 2 days, and the immature DCs developed into mature DCs (8). Then, cells were incubated with FITC-CD1a (1:40; Bioscience, 11-0019-42), FITC-CD83 (1:40; Bioscience, 11-0839-42) and FITC-CD86 (1:40; Bioscience, MHCD8601) in $4^{\circ} \mathrm{C}$ for $30 \mathrm{~min}$. Flow cytometry analysis (FlowJo v7.6.1, FlowJo LLC) was used to quantify the biomarkers CD1a, CD83 and CD86, while a population of DCs matured. Not all cells in the study can be classed as mature DCs.

Measurement of intracellular ROS. Intracellular ROS production was detected using a ROS assay kit (Beyotime Institute of Biotechnology). Cells were treated as described above. In addition, after cells were treated with $\mathrm{HG}+30 \mathrm{mM}$ dioscin for $24 \mathrm{~h}$, the culture medium was changed to one containing only $30 \mathrm{mM} \mathrm{HG}$ and then cultured for another $24 \mathrm{~h}(\mathrm{HG}+$ Dioscin $+\mathrm{HG}$ group). The DCs were incubated at $37^{\circ} \mathrm{C}$ with $10 \mu \mathrm{mol} / 1$ 2,7'-dichlorodihydrofluorescein diacetate (DCFH-DA) for $20 \mathrm{~min}$ and mixed once every 3-5 $\mathrm{min}$. Then, the cells were washed 3 times using cell culture medium without serum to remove the DCFH-DA that had not entered the cells. Fluorescence intensity was measured at an excitation wavelength of $488 \mathrm{~nm}$ and an emission wavelength of $525 \mathrm{~nm}$ by a TECAN GENios Pro multifunction microplate reader (Tecan Group, Ltd.). The amount of oxygen radicals in each group was expressed as the fluorescence intensity with respect to the control group.

Quantitative (q)PCR. Total RNA was isolated and treated with DNase using TRIzol reagent (Thermo Fisher Scientific, Inc.). The primer sequences of different scavenger receptors, including class A scavenger receptors (SR-A), CD36 and lectin-like oxidized low-density lipoprotein receptor-1 (LOX-1) in DCs were the same as those used in a previous study (8). SR-A was amplified using the sense primer 5'-TCC TCGTGTTTGCAGTTCTC-3' and antisense primer 5'-GCA ATTCTTCGTTTCCCACT-3'. CD36 was amplified using the sense primer 5'-CGCTGAGGACAACACAGTCT-3' and antisense primer 5'-GTTGTCAGCCTCTGTTCCAA-3'. LOX-1 was amplified using the sense primer 5'-GGGCTCATTTAA CTGGGAAA-3' and antisense primer 5'-GAAATTGCTTGC TGGATGAA-3'. $\beta$-actin was amplified using the sense primer 5'-CTCCATCCTGGCCTCGCTGT-3' and antisense primer 5'-GCTGTCACCTTCACCGTTCC-3'. Total RNA was reverse transcribed according to the manufacturer's protocols using a ReverTra Ace- $\alpha$-First Strand cDNA Synthesis kit (Toyobo Life Science). Fluorescence qPCR was performed with SYBR Green Real-Time PCR master mix (SinoBio Biotech) using a CFX96 real time system (Bio-Rad Laboratories, Inc.). The reaction program included $94^{\circ} \mathrm{C}$ for $5 \mathrm{~min}$ and 40 cycles of $94^{\circ} \mathrm{C}$ for $30 \mathrm{sec}, 57^{\circ} \mathrm{C}$ for $30 \mathrm{sec}$, and $72^{\circ} \mathrm{C}$ for $30 \mathrm{sec}$. Relative gene expression was analyzed using the delta-delta threshold cycle (CT) method (28). The system software was used to analyze the gene expression, and the standard curve method was used to determine the copies of each mRNA molecule. The internal reference housekeeping gene $\beta$-actin was used to normalize the expression.

Western blot analysis. DCs were treated with $\mathrm{HG}$ or $\mathrm{HG}+$ $30 \mathrm{mM}$ dioscin. Then, the cells were washed with ice-cold PBS and extracted in cold cell lysis buffer (Beyotime Institute of Biotechnology). The bicinchoninic acid protein assay reagent kit (Beyotime Institute of Biotechnology) was used to measure the protein concentration. SDS-PAGE (8\%) was used to fractionate the proteins $(20 \mu \mathrm{g})$ in each experimental group, and they were then transferred to nitrocellulose membranes (EMD Millipore) and blocked with 5\% nonfat 
milk for $1 \mathrm{~h}$ at room temperature. The primary antibodies rabbit polyclonal anti-p38 MAPK (p38; 1:1,000; Cell Signaling Technology, Inc., 9212), rabbit polyclonal anti-p-p38 MAPK (Thr180/Tyr182; pp38; 1:500; Cell Signaling Technology, Inc., 4631), and monoclonal mouse anti- $\alpha$-tubulin (1:1,000; Sigma Aldrich, T6199) were added and incubated for $12 \mathrm{~h}$ at $4^{\circ} \mathrm{C}$. The goat polyclonal anti-mouse IgG-HRP (1:1,000; Santa Cruz Biotechnology, Inc., sc-2005) and anti-rabbit IgG-HRP (1:1,000; Santa Cruz Biotechnology, Inc., sc-2004) were used as secondary antibodies, and incubated for $2 \mathrm{~h}$ at room temperature. Steptavidin-HRP (Beyotime Institute of Biotechnology) was added and incubated for $2 \mathrm{~h}$ at $37^{\circ} \mathrm{C}$ and then an ECL detection system (Thermo Fisher Scientific, Inc.) was used to detect antigen-antibody complexes according to the manufacturer's protocol. The resulting bands were analyzed using ImageQuant LAS 4000 (GE Healthcare Life Sciences). All values were normalized to the tubulin loading control.

ELISA analysis. DCs were incubated with $\mathrm{HG}$ or $\mathrm{HG}+$ dioscin for $24 \mathrm{~h}$, and the total proteins were extracted with extraction solution [200 $\mu 15 \mathrm{M}$ guanidine $\mathrm{HCl}, 50 \mathrm{mM}$ Tris- $\mathrm{HCl}$ (pH 8.0) and protease inhibitor] and then centrifuged at $4^{\circ} \mathrm{C}$ at $12,000 \mathrm{x} \mathrm{g}$ for $30 \mathrm{~min}$ to remove insoluble materials. Levels of IL-6, IL-10, and IL-12 were measured using IL-6, IL-10 and IL-12 ELISA kits (R\&D Systems, Inc, D6050, D1000B and D1200), respectively.

oxLDL uptake. DCs were incubated with $10 \mu \mathrm{g} / \mathrm{ml}$ Dil-oxLDL (Beijing Union-Biology Co. Ltd.) for $60 \mathrm{~min}$ at $37^{\circ} \mathrm{C}$ after treatment with 5.5 or $30 \mathrm{mmol} / \mathrm{l}$ glucose for $24 \mathrm{~h}$. Then, cells were harvested and washed with PBS three times at $4^{\circ} \mathrm{C}$. Flow cytometry analysis (FlowJo v7.6.1, FlowJo LLC) was used to quantify the Dil-oxLDL-incorporated fraction.

Statistical analysis. All of the experiments on cells were repeated three times. Data were expressed as the mean $\pm \mathrm{SD}$. One-way ANOVA followed by the Tukey-Kramer post hoc test (version 17.0; SPSS Inc.) was used to determine significant differences. $\mathrm{P}<0.05$ was considered statistically significant.

\section{Results}

Mature DCs. The mature DCs had a typical treelike shape (Fig. 1A). Costimulatory molecules, antigen-presenting molecules and adherence factors, including human leukocyte antigen, CD1a, CD80, CD83 and CD86, have been shown to be upregulated in mature DCs (29-32). In the current study, the expression of CD1a, CD83 and CD86 was observed in mature DCs. However, a number of, but not all, DCs showed signs of maturation (Fig. 1B-D).

Measurement of ROS production. The effect of dioscin on the production of ROS in DCs is shown in Fig. 2. Compared with the levels in the control group, HG induced the formation of ROS in human monocyte-derived DCs $(\mathrm{P}<0.01)$. Compared with the levels in the HG group, 20-40 $\mathrm{mM}$ dioscin inhibited ROS production in $\mathrm{HG}$ conditions $(\mathrm{P}<0.05)$. Moreover, the inhibitory effect of dioscin at $30 \mathrm{mM}$ and $40 \mathrm{mM}$ was similar; thus, $30 \mathrm{mM}$ dioscin was selected as the reference concentration for subsequent experiments. In addition, the ROS production in the $\mathrm{HG}+$ Dioscin $+\mathrm{HG}$ group was similar to those of cells treated only with $30 \mathrm{mM}$ HG in the HG group ( $\mathrm{P}>0.05$; Fig. S1).

Effect of dioscin on scavenger receptor expression. The effects of dioscin on the expression of SR-A, CD36 and LOX-1 are shown in Fig. 3A-C. Compared with the expression in the control group, the expression of SR-A, CD36 and LOX-1 mRNA was significantly increased in the HG group and HG + dioscin group $(\mathrm{P}<0.05)$. Nevertheless, dioscin treatment led to a significant reduction in SR-A, CD36 and LOX-1 mRNA expression compared with that of the HG group $(\mathrm{P}<0.01)$. The expression levels of the scavenger receptors SR-A, CD36 and LOX-1 in the HG + Dioscin + HG group were similar to those of cells treated only with $30 \mathrm{mM} \mathrm{HG}$ in $\mathrm{HG}$ group ( $\mathrm{P}>0.05$; Fig. S2).

Effect of dioscin on p38 MAPK protein levels. The effect of dioscin on the protein level of p38 MAPK is shown in Fig. 3D and E. Compared with the level in the control group, the protein level of p38 MAPK was increased significantly in the HG group ( $\mathrm{P}=0.006)$. Nevertheless, dioscin treatment led to a significant reduction in the $\mathrm{p} 38$ MAPK protein level compared with that of the HG group $(\mathrm{P}=0.015)$.

Effect of dioscin on DC cytokine secretion. The effects of dioscin on the cytokine secretion of IL-6, IL-10 and IL-12 are shown in Fig. 3F-H. Compared with the levels of the control group, the levels of IL- 6 and IL-12 were markedly increased in the HG group, whereas HG significantly reduced the IL-10 level $(\mathrm{P}<0.05)$. Furthermore, when compared with the levels of the HG group, the levels of IL-6 and IL-12 were significantly reduced, and the IL-10 level was significantly elevated in the $\mathrm{HG}+$ dioscin group $(\mathrm{P}<0.05)$.

Effect of dioscin on the oxLDL uptake by DCs. Compared with the uptake in the control group, the oxLDL uptake by DCs was markedly increased in the HG group $(\mathrm{P}<0.05)$. However, when compared with the uptake in the HG group, the oxLDL uptake by DCs was significantly reduced in the $\mathrm{HG}+$ dioscin group $(\mathrm{P}<0.05)$, as shown in Fig. 4.

\section{Discussion}

Type 2 diabetes has become a major public health problem and may lead to a number of severe complications, including: Diabetic nephropathy; diabetic eye complications; diabetic foot; diabetic cardiovascular complications; diabetic cerebrovascular disease; and diabetic neuropathy $(1,2)$. The major cause of type 2 diabetes is the disturbance of carbohydrate metabolism and insulin resistance (3). Current drug therapies for diabetes include sulfonylurea, biguanide, euglycemic agent, $\alpha$-glucosidase inhibitors, food glucose regulators and dipeptidyl peptidase-4 inhibitors (33). However, these treatments have various side effects, including weight gain, hypoglycemia and drug resistance, with high rates of secondary failure (33). Therefore, it is necessary to discover new antidiabetic drugs with higher curative effects, but fewer side effects. Recently, a variety of natural compounds have been shown to have effects on type 2 diabetes through various mechanisms, such 

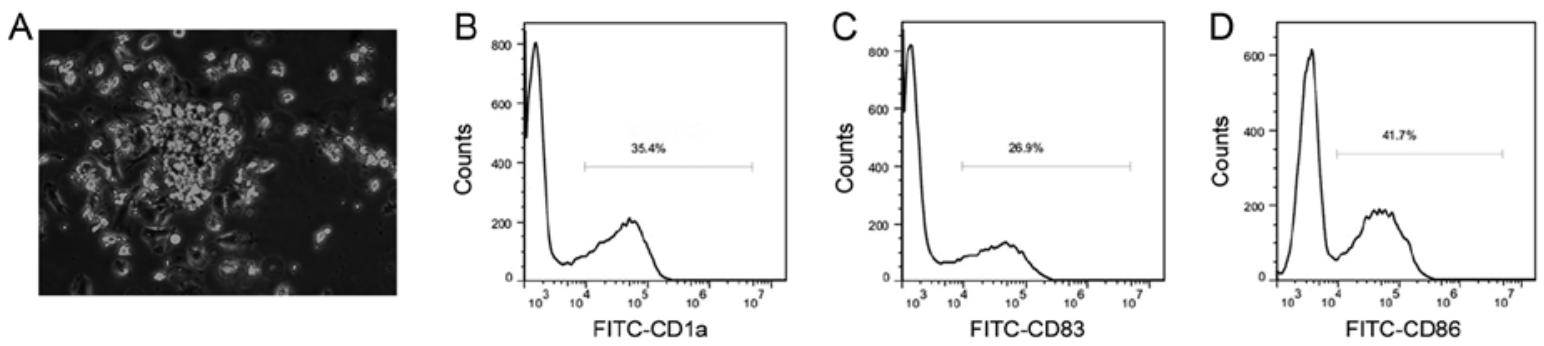

Figure 1. Characteristics of mature DCs. (A) Images of mature DCs with typical treelike shape (magnification, x200). Expression of (B) CD1a, (C) CD83 and (D) CD86.DCs, dendritic cells.

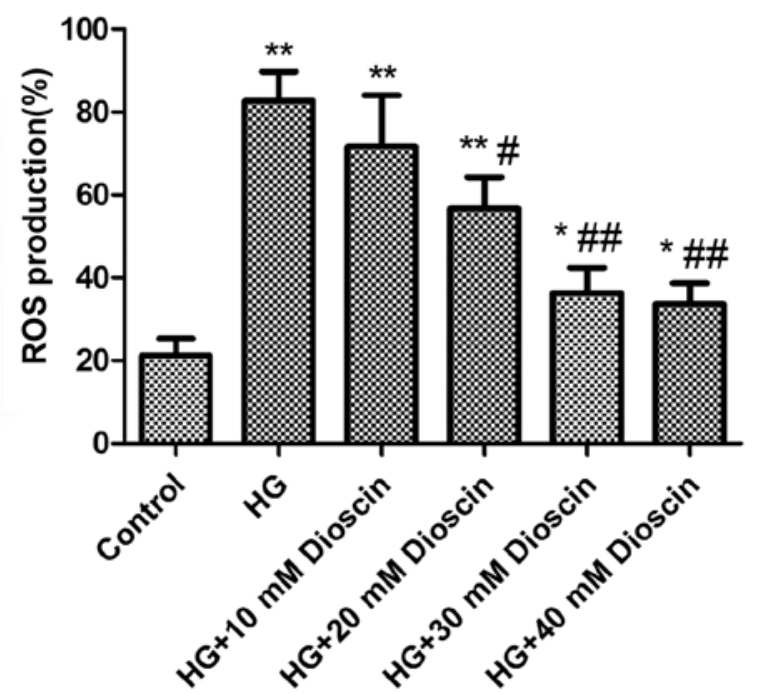

Figure 2. Effect of dioscin on ROS production by DCs. HG induced the formation of ROS in DCs, while 20-40 mM dioscin inhibited ROS production in HG conditions. ${ }^{*} \mathrm{P}<0.05,{ }^{* *} \mathrm{P}<0.01$ vs. control group; ${ }^{\#} \mathrm{P}<0.05,{ }^{\# \#} \mathrm{P}<0.01$ vs. HG group. ROS, reactive oxygen species; DCs, dendritic cells; HG, high glucose.

as flavonoids, polyphenols, terpenoids, alkaloids, saponins and quinones (34).

Dioscin is a natural product that has been shown to have beneficial effects on the inhibition of ischemic stroke-induced inflammation and protective effects against doxorubicin-induced cardiotoxicity. It has also been shown to inhibit colon cancer cell growth through the inhibition of inflammation, oxidative stress and apoptosis in previous studies (24,35-37). A previous study showed that the total saponins from Dioscorea nipponica Makino, including dioscin, could significantly reduce blood glucose levels, improve the levels of oral glucose and insulin tolerance, and increase body weight and serum insulin levels via the regulation of MAPKs, NF- $\kappa \mathrm{B}$, peroxisome proliferator-activated receptor $\gamma$ and glycogen synthase kinase-3 $\beta$ pathways (38). Wei et al (39) reported that dioscin may prevent myocardial injury in diabetic rats by upregulating the nitric oxide-soluble guanylyl cyclase-cGMP-protein kinase G pathway. Dioscin also attenuated high-fat diet-induced insulin resistance of adipose tissue by regulating the insulin receptor substrate-1/PI3K/Akt signaling pathway in an animal model (40). DCs are antigen-presenting cells that can activate $T$ cells and stimulate vascular inflammation in the process of atherosclerosis, thus playing an important role in its development (41-43). In the present study it was hypothesized that dioscin may have some effects on DCs.

Only mature DCs can play a role in the immune reaction. oxLDL can induce the maturation of DCs $(8,11-12)$. In the current study, it was found that under $\mathrm{HG}$, the expression of scavenger receptors, including SR-A, CD36 and LOX-1, was upregulated, as well as oxLDL uptake. Scavenger receptor upregulation and oxLDL uptake are considered critical steps in the initiation and progression of atherosclerosis in diabetes patients $(8,11)$. Dioscin could decrease the expression of the three scavenger receptors and oxLDL uptake, thereby preventing DC maturation and inhibiting atherosclerosis in patients with diabetes in the early stage of its development.

Dioscin showed anti-liver fibrosis effects via Sirt1/nuclear related factor 2-mediated inhibition of the p38 MAPK pathway (44). Under HG conditions, the production of intracellular ROS was increased and the p38 MAPK pathway was activated, upregulating the expression of scavenger receptors and subsequently activating the $\mathrm{NF}-\kappa \mathrm{B}$ pathway (45). Glucose-induced oxidative stress led to the activation of $\mathrm{p} 38$ MAPK in macrophages and DCs $(8,46)$, which can lead to oxLDL uptake, and oxLDL-presenting DCs can activate naive T-cells to induce the inflammatory cascade and promote the formation of atherosclerotic plaques $(8,11)$. In the current study, it was observed that dioscin partially reversed p38 MAPK protein upregulation in HG conditions. However, only one protein was observed and it was not at mRNA level, which is a limitation of this work.

$\mathrm{Hu}$ et al (15) reported that dioscin reduced ROS levels caused by hypoxia-reoxygenation injury in IEC- 6 cells and showed a protective effect against II/R injury by modulating the miR-351-5/Sirt6 signal to reduce oxidative stress. Qiao et al (47) reported that dioscin reduced ROS levels in renal tissue and showed protective effects against fructose-induced renal damage by modulating Sirt3-mediated oxidative stress, renal fibrosis, lipid metabolism and inflammation. Dioscin significantly attenuated NRK-52E cell injury and reduced ROS levels in vitro (48). In the present study, it was found that dioscin could reduce ROS generation in DCs under HG conditions. Moreover, there was a significant dose-effect relationship in the inhibition of ROS generation. However, in the study by Lv et al (49), dioscin showed noteworthy anticancer activity in glioblastoma cells by promoting ROS accumulation, inducing DNA damage and activating mitochondrial signaling pathways, which seems contrary to the results of the present study. In human gastric carcinoma MGC-803 cells, dioscin caused DNA damage and mitochondrial changes, and 

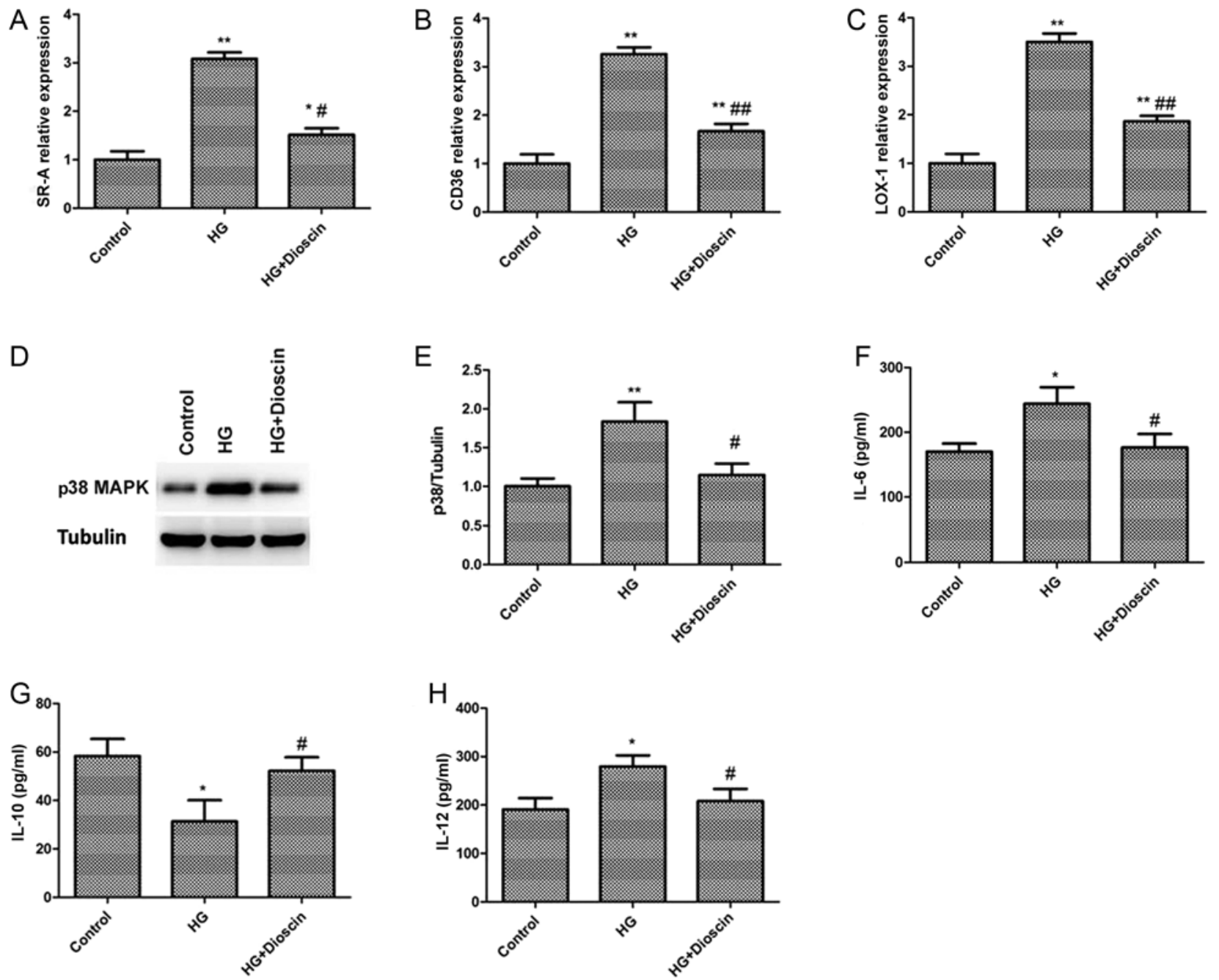

Figure 3. Effect of dioscin on expression of molecules. Bar graphs showing that the upregulation of (A) class A scavenger receptors, (B) CD36 and (C) lectin-like oxidized low-density lipoprotein receptor-1 genes was partially abolished by dioscin. (D) Western blot and (E) bar graph showing that dioscin partially reversed p38 MAPK protein upregulation. Bar graph showing that HG-induced (F) increased secretion of IL-6, (G) decreased secretion of IL-10 and $(\mathrm{H})$ increased secretion of IL-12 in DCs was reversed by dioscin. ${ }^{*} \mathrm{P}<0.05,{ }^{* *} \mathrm{P}<0.01$ vs. control group; ${ }^{\#} \mathrm{P}<0.05$, ${ }^{\# \#} \mathrm{P}<0.01$ vs. HG group. HG, high glucose; MAPK, mitogen-activated protein kinase; DCs, dendritic cells; IL, interleukin.
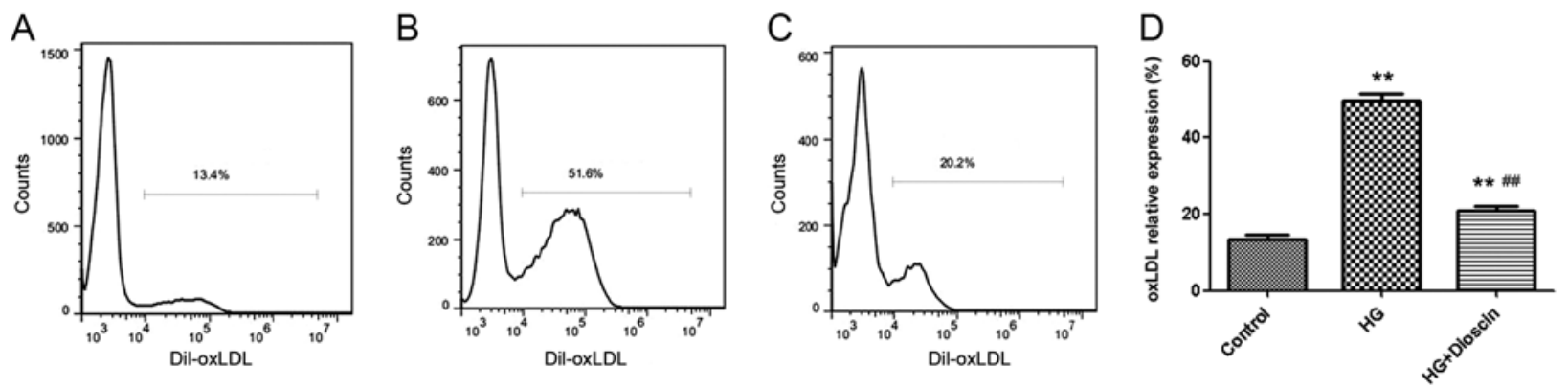

Figure 4. Effect of dioscin on expression of oxLDL. (A) Expression of oxLDL in control group. (B) oxLDL uptake by DCs was markedly increased in the HG group. (C) oxLDL uptake by DCs was significantly reduced in the HG + dioscin group. (D) Statistical analysis of oxLDL uptake by DCs in the three groups. ${ }^{* *} \mathrm{P}<0.01$ vs. control group; ${ }^{\# \#} \mathrm{P}<0.01$ vs. HG group. oxLDL, oxidized low-density lipoprotein; DCs, dendritic cells; HG, high glucose; Dil, diOlistic.

induced ROS generation (50). The reason for the divergence in the effect of dioscin on ROS is not known. In the present study it was hypothesized that the effect of dioscin on ROS generation depends on the cell types. For tumor cells, dioscin promotes ROS accumulation to show anticancer effects; however, for other cell types, dioscin inhibits ROS generation to show protective effects, and the mechanism needs further investigation.

In the presence of $\mathrm{HG}$, the production of proinflammatory cytokines, such as IL-6 and IL-12, was increased in human DCs and then induced DC maturation. In the early stage of type 2 diabetes mellitus, the IL- 6 level was evaluated, and it 
was shown to have a critical role in the development of atherosclerosis (51). In $\mathrm{T}$ helper cell differentiation within the plaque and $\mathrm{T}$ cell recruitment into the plaque, IL-12 appears to play important roles (52). In the current study, it was also discovered that dioscin could reverse the effects of HG-induced increases in the production of IL- 6 and IL-12 by DCs. Considering the role of IL-6 in the formation of atherosclerotic lesions and IL-12 in plaque destabilization, these findings suggested that dioscin potentially inhibits atherosclerosis by weakening DC maturation and immunity. IL-10 is an anti-inflammatory cytokine that can downregulate the inflammatory response and antagonize inflammatory mediators in various inflammatory diseases, including atherosclerosis $(8,53)$, which suggests that dioscin potentially inhibits atherosclerosis by promoting the production of IL-10 in DCs. In addition, the ROS production and expression levels of the scavenger receptors SR-A, CD36 and LOX-1 in HG + Dioscin + HG group were similar to those of cells only treated with $30 \mathrm{mM} \mathrm{HG}$ in HG group. This suggested that the reported effects are reversed when dioscin is removed from cell culture.

In conclusion, this study demonstrated that dioscin could attenuate the production of ROS, inflammatory cytokine secretion and oxLDL uptake by DCs. This is by preventing the expression of scavenger receptors and p38 MAPK, thus having a positive role in preventing atherogenesis.

\section{Acknowledgements}

Not applicable.

\section{Funding}

The present study was funded by: The Joint Medical Research Project of Chongqing Municipal Science and Technology Commission and Municipal Health Commission (grant no. 2018MSXM100); the Chongqing Research Institute Performance Incentive Guidance Special Project (grant no. cstc2018jxj1130038); and the High-level Medical Reserved Personnel Training Project of Chongqing.

\section{Availability of data and materials}

The datasets used and/or analyzed during the present study are available from the corresponding author upon reasonable request.

\section{Authors' contributions}

YiL, YoL and TY performed the experiments; MW designed the study and reviewed the manuscript; YiL analyzed and interpreted the data; MW acquired the data and drafted and edited the manuscript.

\section{Ethics approval and consent to participate}

Healthy volunteers provided informed consent, and the present study was approved by the Ethics Committee of the Chongqing Traditional Chinese Medicine Hospital.

\section{Patient consent for publication}

Not applicable.

\section{Competing interests}

The authors declare that they have no competing interests.

\section{References}

1. Abraham TM, Pencina KM, Pencina MJ and Fox CS: Trends in diabetes incidence: the Framingham heart study. Diabetes Care 38: 482-487, 2015.

2. Geiss LS, Wang J, Cheng YJ, Thompson TJ, Barker L, Li Y, Albright AL and Gregg EW: Prevalence and incidence trends for diagnosed diabetes among adults aged 20 to 79 years, United States, 1980-2012. JAMA 312: 1218-1226, 2014.

3. Kosiborod M, Gomes MB, Nicolucci A, Pocock S, Rathmann W, Shestakova MV, Watada H, Shimomura I, Chen H, Cid-Ruzafa J, et al: Vascular complications in patients with type 2 diabetes: Prevalence and associated factors in 38 countries (the DISCOVER study program). Cardiovasc Diabetol 17: 150, 2018.

4. Suzuki LA, Poot M, Gerrity RG and Bornfeldt KE: Diabetes accelerates smooth muscle accumulation in lesions of atherosclerosis: Lack of direct growth-promoting effects of high glucose levels. Diabetes 50: 851-860, 2001.

5. Gilardini Montani MS, Granato M, Cuomo L, Valia S, Di Renzo L, D'Orazi G, Faggioni A and Cirone M: High glucose and hyperglycemic sera from type 2 diabetic patients impair DC differentiation by inducing ROS and activating Wnt/3-catenin and p38 MAPK. Biochim Biophys Acta 1862: 805-813, 2016.

6. Consolidated guidelines on the use of antiretroviral drugs for treating and preventing HIV infection: Recommendations for a Public Health Approach. 2nd edition, Geneva, 2016.

7. Hansson GK: Inflammation, atherosclerosis, and coronary artery disease. N Engl J Med 352: 1685-1695, 2005.

8. Lu H, Yao K, Huang D, Sun A, Zou Y, Qian J and Ge J: High glucose induces upregulation of scavenger receptors and promotes maturation of dendritic cells. Cardiovasc Diabetol 12: 80, 2013.

9. Ptak W, Klimek M, Bryniarski K, Ptak M and Majcher P: Macrophage function in alloxan diabetic mice: Expression of adhesion molecules, generation of monokines and oxygen and NO radicals. Clin Exp Immunol 114: 13-18, 1998.

10. Beauloye C, Marsin AS, Bertrand L, Krause U, Hardie DG, Vanoverschelde JL and Hue L: Insulin antagonizes AMP-activated protein kinase activation by ischemia or anoxia in rat hearts, without affecting total adenine nucleotides. FEBS Lett 505: 348-352, 2001.

11. Lu H, Huang D, Yao K, Li C, Chang S, Dai Y, Sun A, Zou Y, Qian J and Ge J: Insulin enhances dendritic cell maturation and scavenger receptor-mediated uptake of oxidised low-density lipoprotein. J Diabetes Complications 29: 465-471, 2015.

12. Huang D, Lu H, Liu H, Yao K, Sun A, Zou Y and Ge J: Losartan attenuates human monocyte-derived dendritic cell immune maturation via downregulation of lectin-like oxidized low-density lipoprotein receptor-1. J Cardiovasc Pharmacol 60: 133-139, 2012.

13. Tao X, Yin L, Xu L and Peng J: Dioscin: A diverse acting natural compound with therapeutic potential in metabolic diseases, cancer, inflammation and infections. Pharmacol Res 137: 259-269, 2018.

14. Mao Z, Han X, Chen D, Xu Y, Xu L, Yin L, Sun H, Qi Y, Fang L, Liu $\mathrm{K}$ and Peng J: Potent effects of dioscin against hepatocellular carcinoma through regulating TP53-induced glycolysis and apoptosis regulator (TIGAR)-mediated apoptosis, autophagy, and DNA damage. Br J Pharmacol 176: 919-937, 2019.

15. Hu Y, Mao Z, Xu L, Yin L, Tao X, Tang Z, Qi Y, Sun P and Peng J: Protective effect of dioscin against intestinal ischemia/reperfusion injury via adjusting miR-351-5p-mediated oxidative stress. Pharmacol Res 137: 56-63, 2018.

16. Zheng L, Yin L, Xu L, Qi Y, Li H, Xu Y, Han X, Liu K and Peng J: Protective effect of dioscin against thioacetamide-induced acute liver injury via FXR/AMPK signaling pathway in vivo. Biomed Pharmacother 97: 481-488, 2018.

17. Yao H, Tao X, Xu L, Qi Y, Yin L, Han X, Xu Y, Zheng L and Peng J: Dioscin alleviates non-alcoholic fatty liver disease through adjusting lipid metabolism via SIRT1/AMPK signaling pathway. Pharmacol Res 131: 51-60, 2018.

18. Yao H, Hu C, Yin L, Tao X, Xu L, Qi Y, Han X, Xu Y, Zhao Y, Wang $C$ and Peng J: Dioscin reduces lipopolysaccharide-induced inflammatory liver injury via regulating TLR4/MyD88 signal pathway. Int Immunopharmacol 36: 132-141, 2016. 
19. Yao H, Sun Y, Song S, Qi Y, Tao X, Xu L, Yin L, Han X, Xu Y, $\mathrm{Li} \mathrm{H}$, et al: Protective effects of dioscin against lipopolysaccharide-induced acute lung injury through inhibition of oxidative stress and inflammation. Front Pharmacol 8: 120, 2017.

20. Zhang Y, Tao X, Yin L, Xu L, Xu Y, Qi Y, Han X, Song S, Zhao Y, Lin Y, et al: Protective effects of dioscin against cisplatin-induced nephrotoxicity via the microRNA-34a/sirtuin 1 signalling pathway. Br J Pharmacol 174: 2512-2527, 2017.

21. Zheng L, Han X, Hu Y, Zhao X, Yin L, Xu L, Qi Y, Xu Y, Han X, Liu K and Peng J: Dioscin ameliorates intestinal ischemia/reperfusion injury via adjusting miR-351-5p/MAPK13-mediated inflammation and apoptosis. Pharmacol Res 139: 431-439, 2019.

22. Tao X, Xu L, Yin L, Han X, Qi Y, Xu Y, Song S, Zhao Y and Peng J: Dioscin induces prostate cancer cell apoptosis through activation of estrogen receptor- $\beta$. Cell Death Dis 8: e2989, 2017.

23. Si L, Xu L, Yin L, Qi Y, Han X, Xu Y, Zhao Y, Liu K and Peng J: Potent effects of dioscin against pancreatic cancer via miR-149-3P-mediated inhibition of the Akt1 signalling pathway. Br J Pharmacol 174: 553-568, 2017.

24. Zhao L, Tao X, Qi Y, Xu L, Yin L and Peng J: Protective effect of dioscin against doxorubicin-induced cardiotoxicity via adjusting microRNA-140-5p-mediated myocardial oxidative stress. Redox Biol 16: 189-198, 2018.

25. Wu S, Xu H, Peng J, Wang C, Jin Y, Liu K, Sun H and Qin J: Potent anti-inflammatory effect of dioscin mediated by suppression of TNF- $\alpha$-induced VCAM-1, ICAM-1and EL expression via the NF- $\kappa$ B pathway. Biochimie 110: 62-72, 2015

26. Liu M, Xu L, Yin L, Qi Y, Xu Y, Han X, Zhao Y, Sun H, Yao J Lin Y, et al: Potent effects of dioscin against obesity in mice. Sci Rep 5: 7973, 2015.

27. Tao X, Qi Y, Xu L, Yin L, Han X, Xu Y, Wang C, Sun H and Peng J: Dioscin reduces ovariectomy-induced bone loss by enhancing osteoblastogenesis and inhibiting osteoclastogenesis. Pharmacol Res 108: 90-101, 2016.

28. Livak KJ and Schmittgen TD: Analysis of relative gene expression data using real-time quantitative PCR and the 2(-Delta Delta C(T)) method. Methods 25: 402-408, 2001.

29. Coventry BJ, Austyn JM, Chryssidis S, Hankins D and Harris A Identification and isolation of CD1a positive putative tumour infiltrating dendritic cells in human breast cancer. Adv Exp Med Biol 417: 571-577, 1997.

30. Zhang LF, Okuma K, Tanaka R, Kodama A, Kondo K, Ansari AA and Tanaka Y: Generation of mature dendritic cells with unique phenotype and function by in vitro short-term culture of human monocytes in the presence of interleukin- 4 and interferon-beta. Exp Biol Med (Maywood) 233: 721-731, 2008.

31. Pan $\mathrm{CH}$ and Luo RC: Tumor necrosis factor-alpha pretreatment for in vitro culture of mature dendritic cells. Di Yi Jun Yi Da Xue Xue Bao 23: 114-117, 2003 (In Chinese).

32. Fernandez Pujol B, Lucibello FC, Zuzarte M, Lütjens P, Müller R and Havemann K: Dendritic cells derived from peripheral monocytes express endothelial markers and in the presence of angiogenic growth factors differentiate into endothelial-like cells. Eur J Cell Biol 80: 99-110, 2001

33. Kurosaki $\mathrm{E}$ and Ogasawara $\mathrm{H}$ : Ipragliflozin and other sodium-glucose cotransporter-2 (SGLT2) inhibitors in the treatment of type 2 diabetes: Preclinical and clinical data. Pharmacol Ther 139: 51-59, 2013

34. Xu L, Li Y, Dai Y and Peng J: Natural products for the treatment of type 2 diabetes mellitus: Pharmacology and mechanisms. Pharmacol Res 130: 451-465, 2018.

35. Zhu S, Tang S and Su F: Dioscin inhibits ischemic stroke-induced inflammation through inhibition of the TLR4/MyD88/NF- $\mathrm{BB}$ signaling pathway in a rat model. Mol Med Rep 17: 660-666, 2018.

36. Li S, Cheng B, Hou L, Huang L, Cui Y, Xu D, Shen X and Li S: Dioscin inhibits colon cancer cells' growth by reactive oxygen species-mediated mitochondrial dysfunction and p38 and JNK pathways. Anticancer Drugs 29: 234-242, 2018.
37. Qin J, Kang Y, Xu Z, Zang C, Fang B and Liu X: Dioscin prevents the mitochondrial apoptosis and attenuates oxidative stress in cardiac H9c2 cells. Drug Res (Stuttg) 64: 47-52, 2014.

38. Yu H, Zheng L, Xu L, Yin L, Lin Y, Li H, Liu K and Peng J: Potent effects of the total saponins from Dioscorea nipponica Makino against streptozotocin-induced type 2 diabetes mellitus in rats. Phytother Res 29: 228-240, 2015.

39. Wei Q, Zhu T, Xiao X, Sun L, Zhang Z and Huang T: Dioscin attenuates myocardial damages in diabetic rats maybe by regulating NO-sGC-cGMP-PKG pathway. Ann Clin Lab Sci 49: 97-104, 2019.

40. Li H, Yu L and Zhao C: Dioscin attenuates high-fat diet-induced insulin resistance of adipose tissue through the IRS-1/PI3K/Akt signaling pathway. Mol Med Rep 19: 1230-1237, 2019.

41. Fang Z, Deng Q, Hu H, Wang X, Sun X, Ge X and Wang P: Characteristics of immunogenic and tolerogenic dendritic cells within the arterial wall in atherosclerosis and in vitro. Int J Clin Exp Med 7: 4846-4856, 2014.

42. Gil-Pulido J and Zernecke A: Antigen-presenting dendritic cells in atherosclerosis. Eur J Pharmacol 816: 25-31, 2017.

43. Zernecke A: Dendritic cells in atherosclerosis: Evidence in mice and humans. Arterioscler Thromb Vasc Biol 35: 763-770, 2015.

44. Gu L, Tao X, Xu Y, Han X, Qi Y, Xu L, Yin L and Peng J: Dioscin alleviates BDL- and DMN-induced hepatic fibrosis via Sirt1/Nrf2-mediated inhibition of p38 MAPK pathway. Toxicol Appl Pharmacol 292: 19-29, 2016.

45. Vanden Berghe W, Plaisance S, Boone E, De Bosscher K, Schmitz ML, Fiers W and Haegeman G: p38 and extracellular signal-regulated kinase mitogen-activated protein kinase pathways are required for nuclear factor-kappaB p 65 transactivation mediated by tumor necrosis factor. J Biol Chem 273: 3285-3290, 1998.

46. Nishizawa T and Bornfeldt KE: Diabetic vascular disease and the potential role of macrophage glucose metabolism. Ann Med 44: $555-563,2012$.

47. Qiao Y, Xu L, Tao X, Yin L, Qi Y, Xu Y, Han X, Tang Z, Ma X, Liu K and Peng J: Protective effects of dioscin against fructose-induced renal damage via adjusting Sirt3-mediated oxidative stress, fibrosis, lipid metabolism and inflammation. Toxicol Lett 284: 37-45, 2018.

48. Zhang Y, Xu Y, Qi Y, Xu L, Song S, Yin L, Tao X, Zhen Y, Han X, Ma X, et al: Protective effects of dioscin against doxorubicin-induced nephrotoxicity via adjusting FXR-mediated oxidative stress and inflammation. Toxicology 378: 53-64, 2017.

49. Lv L, Zheng L, Dong D, Xu L, Yin L, Xu Y, Qi Y, Han X and Peng J: Dioscin, a natural steroid saponin, induces apoptosis and DNA damage through reactive oxygen species: A potential new drug for treatment of glioblastoma multiforme. Food Chem Toxicol 59: 657-669, 2013.

50. Zhao X, Xu L, Zheng L, Yin L, Qi Y, Han X, Xu Y and Peng J: Potent effects of dioscin against gastric cancer in vitro and in vivo. Phytomedicine 23: 274-282, 2016.

51. Maier W, Altwegg LA, Corti R, Gay S, Hersberger M, Maly FE, Sütsch G, Roffi M, Neidhart M, Eberli FR, et al: Inflammatory markers at the site of ruptured plaque in acute myocardial infarction: Locally increased interleukin- 6 and serum amyloid A but decreased C-reactive protein. Circulation 111: 1355-1361, 2005.

52. Zhang X, Niessner A, Nakajima T, Ma-Krupa W, Kopecky SL, Frye RL, Goronzy JJ and Weyand CM: Interleukin 12 induces T-cell recruitment into the atherosclerotic plaque. Circ Res 98: 524-531, 2006

53. Yin Y, Liu W, Ji G and Dai Y: The essential role of p38 MAPK in mediating the interplay of oxLDL and IL-10 in regulating endothelial cell apoptosis. Eur J Cell Biol 92: 150-159, 2013.

This work is licensed under a Creative Commons Attribution-NonCommercial-NoDerivatives 4.0 International (CC BY-NC-ND 4.0) License. 